\title{
Collision Prediction based Genetic Network Programming- Reinforcement Learning for Mobile Robot Navigation in Unknown Dynamic Environments
}

\begin{abstract}
Ahmed H. M. Findi ${ }^{\dagger}$, Mohammad H. Marhaban*, Raja Kamil* and Mohd Khair Hassan*
Abstract - The problem of determining a smooth and collision-free path with maximum possible speed for a Mobile Robot (MR) which is chasing a moving target in a dynamic environment is addressed in this paper. Genetic Network Programming with Reinforcement Learning (GNP-RL) has several important features over other evolutionary algorithms such as it combines offline and online learning on the one hand, and it combines diversified and intensified search on the other hand, but it was used in solving the problem of MR navigation in static environment only. This paper presents GNP-RL based on predicting collision positions as a first attempt to apply it for MR navigation in dynamic environment. The combination between features of the proposed collision prediction and that of GNP-RL provides safe navigation (effective obstacle avoidance) in dynamic environment, smooth movement, and reducing the obstacle avoidance latency time. Simulation in dynamic environment is used to evaluate the performance of collision prediction based GNP-RL compared with that of two state-of-the art navigation approaches, namely, Q-Learning (QL) and Artificial Potential Field (APF). The simulation results show that the proposed GNP-RL outperforms both QL and APF in terms of smooth movement and safer navigation. In addition, it outperforms APF in terms of preserving maximum possible speed during obstacle avoidance.
\end{abstract}

Keywords: Collision prediction, Genetic NETWORK PROGRAMMING with Reinforcement Learning (GNP-RL), Mobile robot navigation, Unknown dynamic environment.

\section{Introduction}

Mobile Robot (MR) navigation has attracted much attention from researchers. The research on navigation in static environment is already matured [1], but that in dynamic environment, which is crucial for many real world applications, has recently received substantial attention [2, 3]. The goals of navigation are obstacle avoidance and navigation time reduction. Nevertheless, another important feature that should be included during navigation is minimizing MR steering angle during obstacle avoidance while providing maximum possible speed to preserve MR smooth path and to avoid big changes in speed set point.

Fuzzy logic controller (FLC) [4-8] is widely used in MR navigation because it is able to emulate human reasoning capabilities of dealing with uncertainties [9]. However, the design of a fuzzy controller is difficult and highly time consuming task, as there are lots of parameters values to define [10]. Therefore, many evolutionary algorithms [1113] were applied to tune the parameters of FLC. The main advantage of these methods is the designers may not need to have a complete knowledge of the problem to be solved.

$\dagger$ Corresponding Author: Control and Systems Engineering Department, University of Technology, Baghdad, Iraq.

(ahmfindi@gmail.com)

* Department of Electrical and Electronic Engineering, Faculty of Engineering, Universiti Putra Malaysia, Malaysia.

(\{mhm, kamil, khair\}@upm.edu.my)

Received: June 27, 2015; Accepted: October 12, 2016
Moreover, the entire optimization process is normally carried out off-line and once trained, the FLC might be suitable for on-line implementations [11, 12]. However the effectiveness of these approaches was studied in point-topoint navigation in dynamic environment where target is static and predefined. On the other hand, machine learning algorithms [14-16] were proposed to obtain time-optimal and collision free path of a MR during its navigation in dynamic environment. However, a precise input/output dataset should be provided for training process of these algorithms, but obtaining the dataset is hard or sometimes is impossible [17]. Instead, reinforcement learning techniques $[8,18]$ are used to learn MR by its online interaction with the environment without the need to provide a dataset.

Artificial potential field (APF) $[6,14,19-24]$ is one of the most commonly used schemes in the field of MR navigation [18]. In the work of $\mathrm{Ge}$ and Cui [20], the authors apply APF in a dynamic environment containing a moving target. In this work, it is assumed that the movement of MR is affected by two potential field forces; the attractive force and repulsive force. Consequently, MR moves under the effect of the sum of these two forces. However, APF suffers from local minima problem [14]. In addition, preserving smooth path during obstacle avoidance has not been considered in the literatures.

The works in [2, 25-27] presented virtual plane, 
generalized velocity obstacle, reactive control design and temporal fuzzy rules, respectively, to allow a MR to avoid dynamic obstacles. However all these techniques are based on measuring the velocities of moving obstacles. Practically, this measurement is noisy and difficult to obtain $[25,28]$.

In 2011, Jaradat et al. [18] presented a smart definition of Q-learning states to navigate MR in an unknown dynamic environment with a moving target. However, this design provides only a perception of an environment without any prediction to the collision positions with obstacles, turns MR strictly $( \pm \pi / 4)$ to avoid obstacles, and moves MR always in constant speed Hereafter, this methodology is referred as Obstacle Target Correlation based Q-Learning (OTCQL).

GNP-RL [29-34] is an extension of GNP [35] and it is efficiently combined evolution and learning. Evolutionary computation generally has an advantage in diversified search ability, while reinforcement learning has an advantage in intensified search ability and online learning. Up to our knowledge, GNP-RL has been used in solving the problem of MR navigation in static environment only. Thus, this paper presents the first attempt to apply GNP-RL to control navigation of MR in dynamic environment.

In this paper, a novel GNP-RL design is presented based on predicting the collision position between MR and static or dynamic obstacles. This proposed design aims to obtain effective obstacle avoidance using minimum changes in steering angle between the consecutive steps to smoothen path trajectory of MR and finding maximum possible speed that is sufficient to exceed obstacle successfully without enforcing a high slowness on MR speed. For this purpose, we propose Collision Prediction environment representation which has the capability of predicting the position of collision based on the positions of target and obstacles without the need for reading their velocities as well as it provides a perception of the current situation of the MR surrounding environment. The proposed design is dedicated to address the obstacle avoidance problem of one obstacle at a time. The main objectives of this paper are:

- Safe navigation in dynamic environment containing a combination of static and dynamic obstacles during chasing a moving target.

- Finding the minimum steering angle changes between consecutive steps of MR during obstacle avoidance in such a way that MR can move smoothly without using sharp turning.

- Control the speed of MR during obstacle avoidance to compromise between maintaining the maximum possible speed and avoiding obstacle.

- Integrating collision prediction and GNP-RL to earn benefits of both the predicting of collision occurrence and features of GNP-RL and to apply it for MR navigation in dynamic environment.

\section{The Proposed Environment Representation}

The objective of this section is to represent unknown dynamic environment surrounding MR at any instant of navigation period by a number of variables. These variables give a considerable perception of the surrounding environment and a prediction of collision occurrence if it exists, in such a way that MR can navigate safely without colliding with any obstacle until catching a moving target. Fig. 1 shows the environment, which contains static and dynamic obstacles, surrounding MR during its navigation toward the moving target.

\subsection{Definitions and Assumptions}

The following definitions and assumptions are made.

1. The considered robot $M R\left(M R_{x}, M R_{y}\right)$ is $\mathrm{MR}$, its position at each instance refers to the coordination origin, and its surrounding environment is divided into four regions $(n=1 \sim 4)$.

2. The goal of MR is to catch the target $G\left(G_{x}, G_{y}\right)$, which is continuously moving. The instantaneous coordinates of the target are known to MR all throughout navigation time, without knowing its future trajectory.

3. The environment is unknown and contains many obstacles, which are either static or dynamic. MR deals with one obstacle at a time, and the nearest obstacle to MR is denoted by $O_{i}\left(x_{i}, y_{i}\right)$.

4. The dynamic obstacles $\left(O_{j k}\right)$ are moved randomly and they behaved in a completely unexpected manner, where $j$ is the obstacle number and $k=0,1, \cdots, T, \cdots, m$ is the time instant. Hence, the initial position of $O_{j}$ is $O_{j 0}\left(x_{j 0}, y_{j 0}\right)$, and the next positions during the navigation are: $O_{j 1}\left(x_{j 1}, y_{j 1}\right), \ldots O_{j T}\left(x_{j T}, y_{j T}\right), \ldots, O_{j m}\left(\mathrm{x}_{j m}, \mathrm{y}_{j m}\right)$.

5. MR has a sensory system, which measures the real-time position of the obstacles within a specific range.

6. MR velocity is always greater than that of the target, i.e. $v_{M R}>v_{G}$. Meanwhile, MR velocity is always greater than or equal to that of obstacles i.e. $v_{M R} \geq v_{\gamma}$.

7. $M R$ becomes in non-safe mode if the distance between $M R$ and $O_{i}$ is less than or equal to the safety distance $\left(d_{s}\right)$. Otherwise, it is in safe mode. On the other hand, $M R$ is considered catching $G$ if the distance between $M R$ and $G$ is equal or less than winning distance $\left(d_{w}\right)$.

\subsection{Dynamic obstacles}

Once MR encounters a dynamic obstacle, it perceives its surrounding environment and predicts collision occurrence through two parameters; Region of Obstacle and Region of Collision that are described as follows:

Region of Obstacle $\left(R_{\gamma n}\right)$ : This represents the first parameter of environment representation, which gives a perception of current surrounding environment. At any 


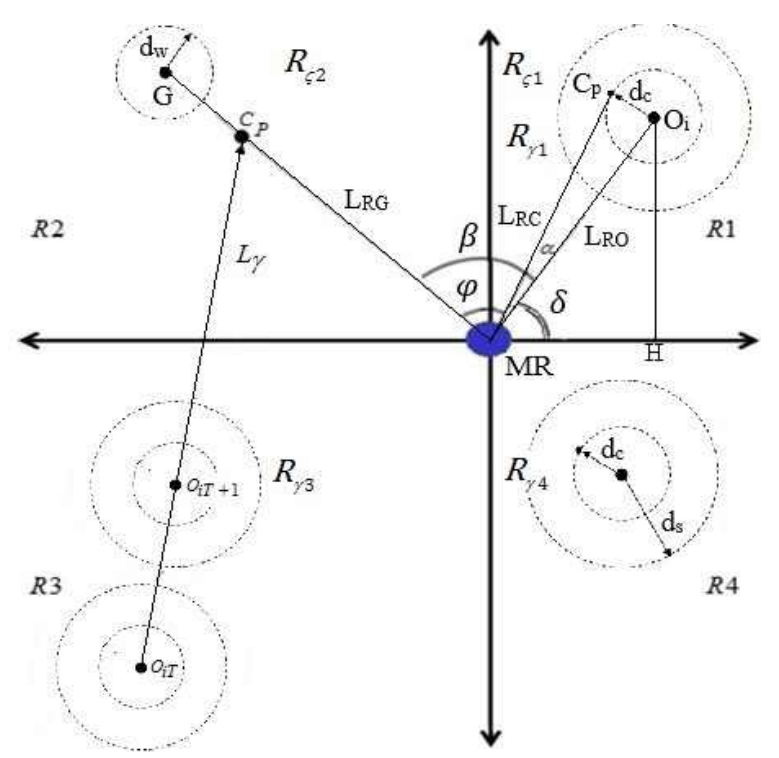

Fig. 1. Graphical representation of collision prediction

instance, the region of $O_{i}$ can be found based on its location related to that of MR. As shown in Fig. 1, $O_{i}$ is located in the third region, $R_{\gamma 3}$, during the two consecutive time instants $T$ and $T+1$.

Region of collision $\left(R_{\varsigma_{n}}\right)$ : This value represents the second parameter of environment representation, which gives prediction of collision position. At each moment of navigation, the predicted point of collision $\left(C_{p}\right)$ is compared with MR position to find the region $\left(R_{\varsigma 1}, R_{\varsigma 2}\right.$, $\left.R_{\varsigma 3}, R_{\varsigma 4}\right)$ of collision. As shown in Fig. $1, C_{p}$ is located in the second region $\left(R_{c^{2}}\right)$,

To predict the existence and position of a collision $C_{p}\left(\mathrm{C}_{x}, \mathrm{C}_{y}\right)$ between MR and a dynamic obstacle, two virtual lines, namely robot line $\left(L_{R G}\right)$ and Obstacle direction Line $\left(L_{\gamma}\right)$, are determined. $L_{R G}$ is the virtual straight line that starts from the MR position and is directed toward the target position. Sampling time is too short compared with the time required to change the direction of a moving obstacle; hence, $O_{i}$ is assumed to be moving in a straight line, starting from $O_{i T}\left(x_{i T}, y_{i T}\right)$, passing through $O_{i T+1}\left(x_{i T+1}, y_{i T+1}\right)$, and continuing in a straight direction along all its future positions. This line is called obstacle direction Line $\left(L_{\gamma}\right)$. Its equation can be determined at each instance based on the last two consecutive positions of $O_{i}$, namely $O_{i T}\left(x_{i T}, y_{i T}\right)$ and $O_{i T+1}\left(x_{i T+1}, y_{i T+1}\right)$.

As shown in Fig. 1, the occurrence of a collision at $C_{p}\left(\mathrm{C}_{x}, \mathrm{C}_{y}\right)$ between $\mathrm{MR}$ and $O_{i}$ is predicted if the following conditions are satisfied: (1) the slopes $\left(m_{R G}\right.$ and $m_{\gamma}$ ) of $L_{R G}$ and $L_{\gamma}$ are unequal, (2) the obstacle moves toward the path of $\mathrm{MR}$, and (3) $C_{p}\left(\mathrm{C}_{x}, \mathrm{C}_{y}\right)$ is located between the positions of $\mathrm{MR}$ and the target. The last condition can be calculated with respect to $\mathrm{x}$-coordinate or $y$-coordinate. However, its calculation for both coordinates is unnecessary because it is bounded by the first two conditions. These three conditions are represented by Eq's.
$1-3$, respectively. Collision is guaranteed to take place if all these conditions are satisfied. However, it will not occur if at least one of these conditions is unsatisfied. This case is called no collision $(N C)$ state. When a collision is predicted, $R_{\varsigma}$ is found by determining the region where $C_{p}\left(\mathrm{C}_{x}, \mathrm{C}_{y}\right)$ is related to the MR position.

$$
\text { Let } d\left(x_{1}, x_{2}\right) \stackrel{\Delta}{=}\left|x_{1}-x_{2}\right| \text { and } B\left(x_{0}, r\right) \stackrel{\Delta}{=}\left\{x \in R \mid d\left(x_{0}, x\right) \leq r\right\}
$$

$$
\begin{array}{lc}
\text { I. Intersection } & m_{R G} \neq m_{\gamma} \\
\text { II. Convergence } & d\left(\mathrm{C}_{p}, \mathrm{O}_{i T+1}\right) \leq d\left(\mathrm{C}_{p}, \mathrm{O}_{i T}\right) \\
\text { III. Boundedness } & C_{x} \in B\left(R G, r+d_{c}\right)
\end{array}
$$

where $B(\mathrm{RG}, \mathrm{r})=\{x \in R \mid d(\mathrm{RG}, \mathrm{x}) \leq \mathrm{r}\}, R G=\left(G_{x}+R_{x}\right) / 2$, $r=d\left(\mathrm{R}_{x}+G_{x}\right) / 2$, and $d_{c}$ is the radius of a virtual circle surrounded each obstacle. This radius represents the summation of the MR radius, obstacle radius, and safety distance.

\subsection{Static Obstacles}

When MR encounters a static obstacle, the same parameters, $R_{\gamma}$ and $R_{\varsigma}$, as in case of dynamic obstacles will be used to represent the surrounding environment. But the difference is only in the way of finding $R_{\varsigma}$. With reference to Fig. 1, we define the following quantities:

1. When MR encounters a static obstacle, i.e. $O_{i T}\left(x_{i T}, y_{i T}\right)=O_{i T+1}\left(x_{i T+1}, y_{i T+1}\right)$, then $R_{\gamma}$ is found as in the case of dynamic obstacles. As shown in Fig. 1, $O_{i}$ is located in the first region $\left(R_{\gamma 1}\right)$.

2. Suppose that the obstacle, $O_{i}$, is surrounding by a virtual circle, which has radius $d_{c}$. The collision is occurred and the execution of that trial is stopped when MR becomes inside or in the circumference of this circle.

3. Three virtual lines and four angles are determined to predict the existence and position of a collision between MR and a static obstacle and then find $R_{\varsigma}$. The virtual lines of robot $\left(\mathrm{L}_{\mathrm{RG}}\right)$, obstacle $\left(\mathrm{L}_{\mathrm{RO}}\right)$, and collision $\left(\mathrm{L}_{\mathrm{RC}}\right)$, are determined. $\mathrm{L}_{\mathrm{RG}}$ is the virtual straight line that starts from MR position and directs toward the target $(\mathrm{G})$ position. $\mathrm{L}_{\mathrm{RO}}$ is the virtual straight line that starts from MR position and directs toward the nearest static obstacle, $O_{i}\left(x_{i}, y_{i}\right) .\left(\mathrm{L}_{\mathrm{RC}}\right)$ is the virtual tangential straight line of the virtual collision circle. This line starts from MR position and directs toward the predictable collision point, $C_{p}\left(\mathrm{C}_{x}, \mathrm{C}_{y}\right)$. On the other hand, four angles $\alpha, \beta, \delta$, and $\phi$ are also determined. $\alpha$ is the angle made by $\mathrm{L}_{\mathrm{RO}}$ and $\mathrm{L}_{\mathrm{RC}}, \beta$ is the angle made by $\mathrm{L}_{\mathrm{RO}}$ and $\mathrm{L}_{\mathrm{RG}}, \delta$ is the angle made by $\mathrm{L}_{\mathrm{RO}}$ and the $\mathrm{x}$-axis, and $\phi$ is the angle made by $\mathrm{L}_{\mathrm{RG}}$ and $\mathrm{L}_{\mathrm{RC}}$.

4. The collision between MR and the static obstacle takes place if $\beta \leq \alpha$. Otherwise, this obstacle will not cause a collision, No Collision, $\mathrm{NC}$, for $\mathrm{MR}$ if it is continued in the same orientation. i.e. 


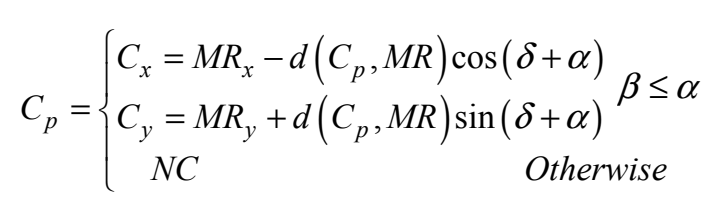

where

$$
\begin{aligned}
\alpha= & \sin ^{-1}\left(d\left(O_{i}, C_{p}\right) / d\left(O_{i}, M R\right)\right), \\
\delta= & \sin ^{-1}\left(d\left(y_{i}, R_{y}\right) / d\left(O_{i}, M R\right)\right), \\
\beta= & \cos ^{-1}\left(\left(d\left(O_{i}, M R\right)^{2}+d(G, M R)^{2}-d\left(G, O_{i}\right)^{2}\right) /\right. \\
& \left.\left(2 d\left(O_{i}, M R\right) d(G, M R)\right)\right) \\
\phi= & \cos ^{-1}\left(\left(d\left(C_{p}, M R\right)^{2}+d(G, M R)^{2}-d\left(G, C_{p}\right)^{2}\right) /\right. \\
& \left.\left(2 d\left(C_{p}, M R\right) d(G, M R)\right)\right) \\
\xi= & \operatorname{sign}\left(R_{x}-x_{i}\right), \psi=\operatorname{sign}(\beta-\phi) \text { and } \\
& \operatorname{sign}(0)=\operatorname{sign}\left(0^{+}\right)=+1
\end{aligned}
$$

5. After calculating the location of the predicted collision, it becomes easy to find in which region, $R_{\varsigma}$, is located corresponding to the position of MR. If $\beta>\alpha$, then it is defined as $N C$ state.

\section{The Proposed GNP-RL}

In this paper, a novel GNP-RL design is presented based on predicting the collision place between MR and a static or dynamic obstacle. This proposed design aims to provide effective obstacle avoidance using minimum steering angle changes so as to smoothen path trajectory of MR and to reach the maximum possible speed that is sufficient to exceed obstacle successfully without the need to high slowness of MR speed.

\subsection{GNP-RL structure}

GNP-RL is constructed of several nodes (node_no ) connected with each other and each node has a unique number ( 0 to node_no-1). These nodes are classified into three types of nodes; start node, judgment node, and processing node as shown in Fig. 2. The judgment nodes and processing nodes are inter-connected to each other by

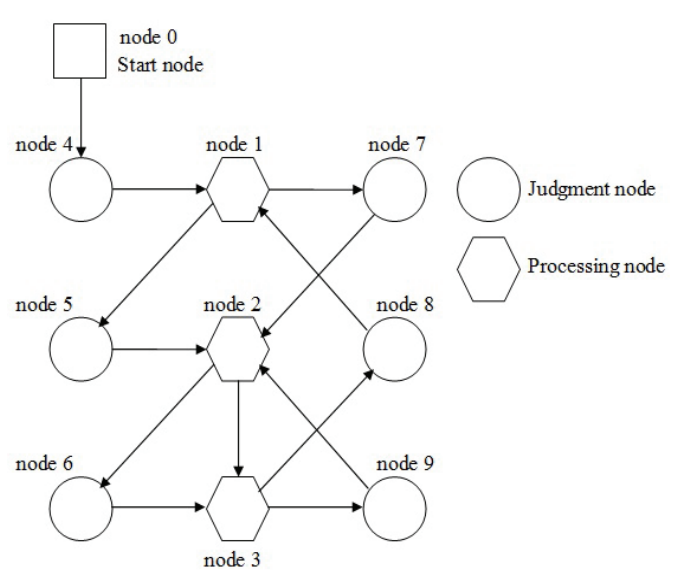

Fig. 2. Basic structure of GNP-RL [29] directed links that indicate the possible transitions from one node to another. The starting node represents the starting point of each node transition and it functions to determine the first node in node transition to be executed. Hence, the first node executed in GNP-RL is determined by the outbound of the starting node which has no inbound connection. . The judgment nodes and processing nodes are inter-connected to each other by directed links that indicate the possible transitions from one node to another. Every judgment node has many outbound connections, so one of these connections is selected according to the if-then decision functions to find the next node to be executed. While the processing nodes have only one outbound connection and they are responsible for taking actions by MR. On the other hand, judgment and processing operations require time to be implemented. This time is called time delay $\left(d_{i}\right)$, which is assigned to each node, and it is participated in evolving flexible and deadlock free programs.

The gene structure of each node is divided into two parts; macro node and sub-node parts as shown in Fig. 3. The macro part consists of node type $\left(N T_{i}\right)$ and time delay spent on executing node $i\left(d_{i}\right)$, where $i$ represents the node number. In this paper, $N T_{i}$ is set to 0,1 , and 2 to represent start, judgment and processing nodes, respectively. $d_{i}$ of judgment nodes is set to one time unit and that of processing nodes is set to five time units. The sub-node part consists of a unique code number $\left(I D_{i k}\right)$ of the judgment/processing, value of judgment $\left(R_{i k}\right)$, value of processing ( $\left.A_{i k p}\right), \mathrm{Q}$ value $\left(Q_{i k p}\right)$ and the number of the next node connected from sub-node $\mathrm{k}$ in node $i\left(C_{i k p}\right)$ where $i k p$ means the parameter $p$ of sub-node $k$ in the node $i, 1 \leq k \leq m_{i}$, and $m_{i}$ is the number of sub-nodes in node $i$.

In this paper, each judgment node is composed of two sub-nodes as shown in Fig. 3. $I D_{i k}$ of judgment nodes represents the regions $R_{\gamma}$ and $R_{\zeta}$. Specifically, $I D_{i k}$ is represented by the values 1 and 2 to refer to $R_{\gamma}$ and $R_{\varsigma}$, respectively. $R_{i k}$ is the value of the region that is identified by $I D_{i k}$. Therefore, the values of $R_{i k}$ can be either 1 to 4 or 1 to 5 depending on the region that is identified by $I D_{i k}$. For example, if the region considered in the sub-node is obstacle region and the obstacle is located in the second region corresponding to the position of $\mathrm{MR}$, then $I D_{i k}=1$ and $R_{i k}=2$. The other parameters of each sub-node are Q-values and connection numbers. Since the value of $R_{\gamma}$ is one of four values and that of $R_{\varsigma}$ is one of five values, either four or five Q-values can be found in every sub-node of judgment nodes depending on the values of $I D_{i k}$ and $R_{i k}$. Similarly for $C_{i k p}$, there are either four or five values in each sub-node of judgment nodes. For processing nodes, each node is composed of two sub-nodes as shown in Fig. 3. $I D_{i k}$ represents the direction of turning, left or right. Specifically, $I D_{i K}$ is represented by the values 3 and 4 to refer to left and right turning, respectively. For each sub-node, $A_{i k p}$ refers to angle of turn and MR speed for $p=1$ or 2 , respectively as 


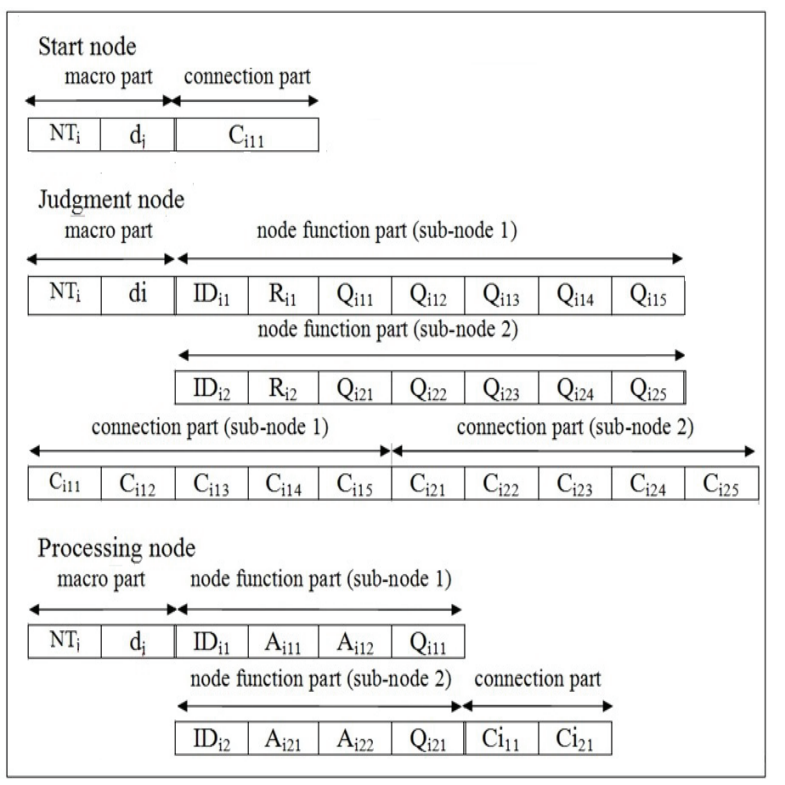

Fig. 3. The gene structure of GNP-RL nodes

well as there is only one Q-value and one $C_{i k p}$. One of the Q-values of the current node is selected by applying $\varepsilon_{-}$ greedy selection method. In this method, the maximum Qvalue among the selected Q-values is chosen with the probability $1-\varepsilon$ or random one is chosen with the probability of $\varepsilon$.

When MR is far away from obstacles $d\left(M R, O_{i}\right)>\mathrm{d}_{s}$, it is considered in safe mode and moved directly to the target. Once MR becomes near an obstacle $d_{c}<d\left(M R, O_{i}\right) \leq \mathrm{d}_{s}$, the controller turns from safe mode to non-safe mode in which GNP-RL guides MR by starting the node transition. This node transition aims to find a suitable processing action through judging current environment state to avoid this obstacle.

The first node executed in the node transition is the start node that guides the transition to one of the judgment nodes. Referring to Fig. 4, if the current node $i$ is a judgment node, the following steps are applied:

1. Calculate $R_{\gamma}$ and $R_{\varsigma}$ as it explained in section 2

2. For each sub-node

2.1 According to the values of $R_{\gamma}$ and $R_{\varsigma}$, specify $I D_{i k}$ and $R_{i k}$ in both sub-nodes.

2.2 Specify the $Q_{i k p}$ and $C_{i k p}$ corresponding to the value of $R_{i k}$.

3. Apply $\mathcal{E}$-greedy selection on the selected Q-values in 2.2 to choose one of them.

4. Select $C_{i k p}$ corresponding to the selected Q-value.

5. Do the node transition according to the selected value $C_{i k p}$.

6. Repeat step (2) if the next node is judgment node. Otherwise, execute the following processing nodes procedure.

The node transitions continues executing the judgment

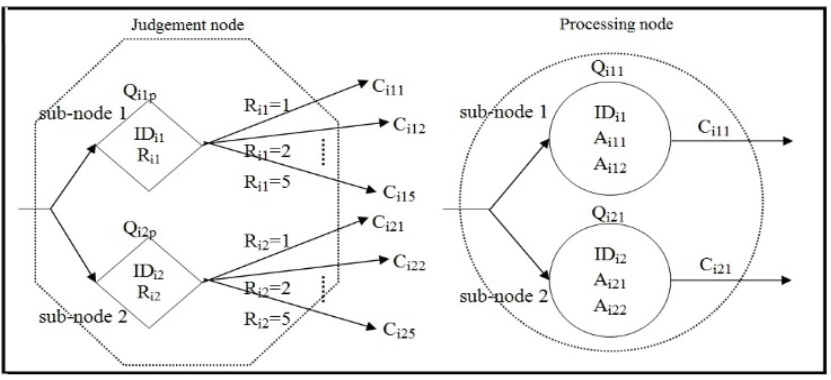

Fig. 4. Node transition

nodes until finding a suitable processing node. To prevent node transition from falling in non-stop loops, the sum of time delay $\left(d_{i}\right)$ of all nodes used in a node transition of one MR step is limited by predefined time unites, named maximum delay of a node transition $\left(d_{\max }\right)$.

The processing node supplies MR by the required degree of the turning angle and speed to avoid the encountered obstacle. Moreover, the selected processing node specifies the next node in GNP-RL which should be executed if the non-safe mode still valid. This process continues until MR avoids the encountered obstacle and changes from non-safe to safe mode.

Referring to Fig. 4, the following steps are applied, if the current node $i$ is a processing node:

(1) Choose the Q-value for each sub-node.

(2) Apply $\varepsilon$-greedy selection on the selected Q-values and choose one of them.

(3) Select the corresponding sub-node according to the selected Q-values.

(4) From the selected sub-node, turns MR to the left or right according to $I D_{i k}$ with angle of turn $A_{i k 1}$ from the virtual line $L_{R G}$, and speed $A_{i k 2}$. Moreover, the next node is specified by $C_{i k 1}$.

(5) Move MR according to the setting values in step 4 and then move the node transition to the node specified by $C_{i k 1}$.

\subsection{Learning and evolution process}

The learning aims to train individuals to find the best node transition of each one by updating the Q-values through State Action Reward State Action (SARSA) [36], while evolution process aims to find the best structure of GNP-RL in population by evolving individuals along all generations. Fig. 5 shows the flowchart of the learning and execution tasks. The first step of learning and evolution process is the initialization of population in which the parameters and values of connections and node functions are set randomly while all Q-values are set to zero. At the end of each generation, the individual which has the highest fitness is chosen as an elite individual and passed directly to the population of the next generation without any modification. The complementary individuals of the next generation are obtained from applying genetic 


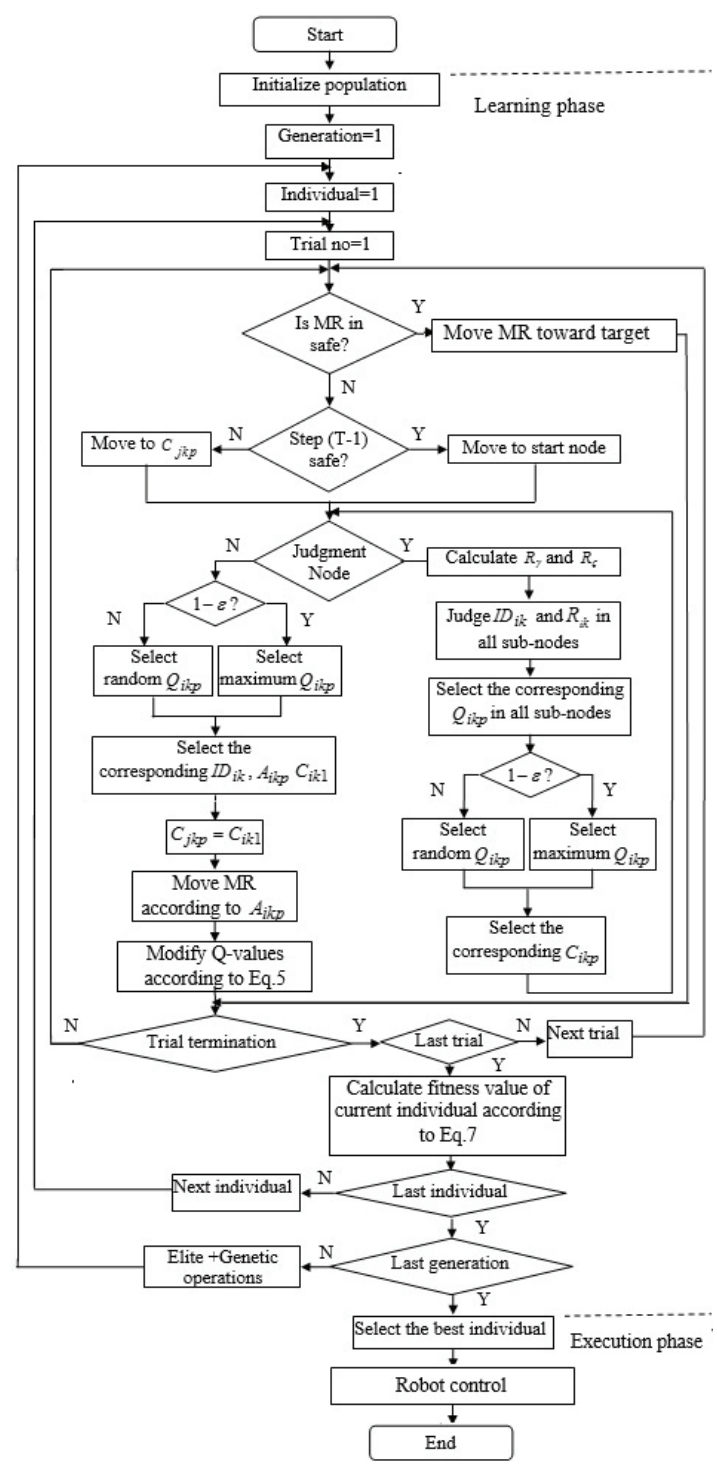

Fig. 5. Flowchart of learning and execution GNP-RL

operations (selection, crossover, and mutation). At the last generation, the individual which provides best performance among all individuals is chosen to be utilized in controlling MR in the testing phase.

\subsection{Q-values update using SARSA}

The node transition of GNP-RL starts once MR encounters an obstacle and it continues until obstacle avoidance takes place. SARSA learning is used to update the Q-values as follows:

1. Suppose the current node is judgment $i$ at time t. Then in every sub-node, GNP-RL refers to all $Q_{i k p}$ and chooses one of them based on the judgment result of $I D_{i k}$ and $R_{i k}$. By applying $\mathcal{E}$-greedy policy, one of these Qvalues is selected.

2. The selected Q-value refers to the selected sub-node. Hence, the corresponding $C_{i k p}$ can be identified which refers in turn to the next node.

3. The node transition is transferred from current node $i$ to next node $j$ at time $\mathrm{t}+1$ and one $Q_{j k p}$ is selected by the same way shown in step 1.

4. Repeat steps 1, 2 and 3 until $C_{i k p}$ refers to a processing node.

5. When the node transition reaches a processing node $j$, GNP-RL refers to all $Q_{j k p}$ and chooses one of them based on $\mathcal{E}$-greedy. Hence, the corresponding $I D_{j k}, A_{j k p}$, and $C_{j k p}$ are selected.

6. Judge MR movement related to the obstacle and get reward $r_{t}$. The calculations of $r_{t}$ will be explained in section 3.5 .

7. The following update is carried out on all nodes along the node transition.

$$
Q_{i k p} \leftarrow Q_{i k p}+\eta\left(r_{t}+\mu Q_{j k p}-Q_{i k p}\right)
$$

where $\eta$ is the learning rate $(0<\eta \leq 1)$ and $\mu$ is the discount rate $(0<\mu \leq 1)$.

\subsection{Genetic operations}

Crossover is executed between two parents generating two offspring. The steps of executing crossover operation can be explained as follows:

1. Select two parents using tournament selection.

2. Each node $i$ in the parents is selected as a crossover node with the probability of Pc.

3. Two parents exchange the genes of the corresponding crossover nodes (i.e., with the same node number).

4. The new generated individuals become the new ones in the next generation.

Mutation is executed on one individual and a new individual is generated. The steps of executing mutation operation can be explained as follows:

1. Select one individual using tournament selection.

2. Mutation operators

2.1. Connection: Each node branch is re-connected to another node with the probability of Pm.

2.2. Function: Each node function (region type in the case of judgment; right/left turning in the case of processing) is changed to another one with the probability of Pm.

2.3. Parameter: Each parameter $A_{i k p}$ in the processing node is changed to another value with the

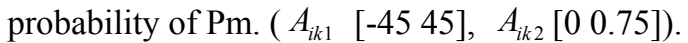

3 . The generated new individual becomes the new one in the next generation [29].

\subsection{Fitness function}

In a dynamic environment with a moving target, MR tries to navigate safely from a start location to a location of 
catching the moving target. During this navigation, MR should find a feasible collision-free path in which the steering angle changes between consecutive steps should be as minimum as possible and the movement should be fast enough to exceed obstacle without collision.

In each generation, every individual in a population has to fulfill obstacle avoidance during target chasing in every trial. A trial ends when either obstacle collision takes place or the time step reaches the predefined time step limit $(=100)$. At every step throughout obstacle avoidance activity, the reward obtained by MR is calculated by Eq. 6 . The reward function is ranged between [0 01$]$ and designed in order to learn obstacle avoidance behavior, that is, MR has to avoid obstacles with trying to make $d\left(M R, O_{i}\right)$ as large as possible where the reward decreases when MR closes to an obstacle and vice versa.

$$
r_{t}=C_{1} e^{C_{2}\left(d\left(M R, O_{i}\right)-d_{s}\right)^{3}}
$$

where $C_{1}=\left\{\begin{array}{cc}0 & d\left(M R, O_{i}\right) \leq d_{c} \\ 1 & \text { otherwise }\end{array}\right.$ and $C_{2}=\left\{\begin{array}{cc}1 & d\left(M R, O_{i}\right) \leq d_{s} \\ 0 & \text { otherwise }\end{array}\right.$.

The fitness function is designed to measure the performance of MR according to its steering angle change and speed. Hence, the fitness function of GNP-RL takes into account both objectives: minimization of steering angle change and maximization of MR speed during obstacle avoidance. The fitness function is calculated by Eq. 7 when all trials of an individual end.

$$
\text { fitness }=\frac{\varpi}{N} \sum_{n=1}^{N} \frac{1}{M} \sum_{m=1}^{M} \frac{C_{3} r_{t}}{e^{r(\Delta \theta)^{2}+\left(v-v_{\max }\right)^{2}}}
$$

where $N$ is the total number of trials, $\mathrm{M}$ is the number of steps required to avoid an obstacle, $r(\Delta \theta)=\frac{\Delta \theta}{\theta_{\max }}=\frac{\theta_{t}-\theta_{t-1}}{\theta_{\max }}$ is the rate of steering angle change between two consecutive steps $(\Delta \theta), v$ is the velocity of the MR, $v_{\max }$ is the maximum velocity of the MR, $C_{3}=\left\{\begin{array}{l}0 d\left(M R, O_{i}\right) \leq d_{c} \\ 5 \quad \text { otherwise }\end{array}\right.$ and $\sigma=\left(\eta_{\text {total }}-\left|\eta_{\text {right }}-\eta_{\text {left }}\right|\right) /$ $\left(\eta_{\text {total }}\right)$ is a factor of balancing the movement of MR in both directions where $\eta_{\text {total }}, \eta_{\text {right }}$, and $\eta_{\text {left }}$ are the total turning actions, number of turnings to right, and number of turning to left during obstacle avoidance in all trials of an individual, respectively. This factor is used to prevent MR from tending to move in one direction to minimize steering angle on the account of safe navigation.

Fig. 6 shows the distribution of the fitness function values when its parameters are changed along all their range where it is assumed that $d\left(M R, O_{i}\right)=1.5 \mathrm{~m}$ and $d_{s}=2 \mathrm{~m}$. It can be noted that the fitness values are increased when steering angle change is decreased and

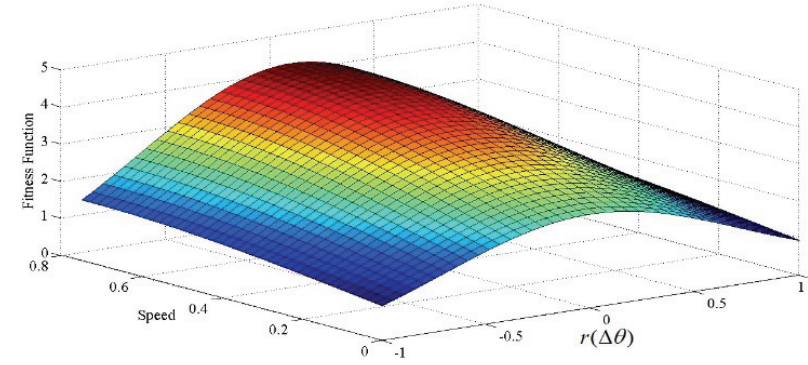

Fig. 6. Fitness function

Table 1. Node functions

\begin{tabular}{c|c|c|c|c|l}
\hline $\begin{array}{c}\text { Node } \\
\text { type }\end{array}$ & $\mathrm{ID}_{\mathrm{ik}}$ & $\mathrm{R}_{\mathrm{ik}}$ & $\mathrm{A}_{\mathrm{ik} 1}$ & $\mathrm{~A}_{\mathrm{ik} 2}$ & Function \\
\hline $\begin{array}{c}\text { Judgment } \\
\text { node }\end{array}$ & 1 & $1, \ldots, 4$ & & & $\begin{array}{l}\text { Judge in which region the } \\
\text { encountered obstacle is } \\
\text { located }\end{array}$ \\
\cline { 2 - 6 } & 2 & $1, \ldots, 5$ & & $\begin{array}{l}\text { Judge in which region the } \\
\text { predicted collision position } \\
\text { is located }\end{array}$ \\
\hline \multirow{2}{*}{$\begin{array}{c}\text { Processing } \\
\text { node }\end{array}$} & 3 & & $0-45^{\circ}$ & $\begin{array}{c}0-0.75 \\
\mathrm{~m} / \mathrm{s}\end{array}$ & $\begin{array}{l}\text { Determine the degree of } \\
\text { turning angle and speed of } \\
\text { MR to the left }\end{array}$ \\
\cline { 2 - 6 } & 4 & & $0-45^{\circ}$ & $\begin{array}{c}0-0.75 \\
\mathrm{~m} / \mathrm{s}\end{array}$ & $\begin{array}{l}\text { Determine the degree of } \\
\text { turning angle and speed of } \\
\text { MR to the right }\end{array}$ \\
\hline
\end{tabular}

Table 2. Simulation parameters

\begin{tabular}{c|l}
\hline Number of individuals & 400(crossover: 299, mutation: 100, elite: 1$)$ \\
\hline Number of nodes & $\begin{array}{l}27(\text { start node: } 1, \text { judgment node: } 16, \\
\text { processing node: } 10)\end{array}$ \\
\hline Parameters of evaluation & Pc-0.1, Pm=0.01, tournament size $=6$ \\
\hline Parameters of learning & $\alpha=0.9, \quad \gamma=0.3, \quad \varepsilon=0.1$ \\
\hline
\end{tabular}

speed is increased. That is, the fitness values go to their maximum value when $r(\Delta \theta)$ approaches to zero and speed approaches to its maximum value.

\subsection{GNP-RL setup}

As shown in Table 1, there are nine judgment functions and two processing functions because $R_{\gamma}$ and $R_{\varsigma}$ have nine states and MR should turn to either left or right to avoid the encountered obstacle. Each processing node sets the degree of turning and speed of MR at $A_{i k 1}$ and $A_{i k 2}$, respectively.

The evolution and learning parameters are shown in Table 2. The number of initial population, crossover probability, mutation probability, tournament size, and maximal offspring generation are set by $400,0.1,0.01,6$, and 6000 , respectively, while the parameters of learning process are set by $\eta=0.9, \mu=0.3$ and $\varepsilon=0.1$. The population in each generation consists of an elite individual, 299 new individuals generated by crossover, and 100 new individuals generated by mutation. Each individual consists of a start node, 16 judgment nodes, and 10 processing nodes. Initially, all the functions and parameters of all individuals are set randomly based on their valid ranges, 
while the $\mathrm{Q}$ values of the sub-nodes are set to zero.

To prevents node transition from falling in non-stop loops, $d_{\max }$ is set to 21 time unites. Hence, the maximum number of nodes that can be used in one node transition cycle is sixteen judgment nodes and one processing node.

\section{Simulation}

The simulation is composed of two phases: learning and execution. The learning phase aims to find an individual that has the ability to achieve the desired safe, smooth, and fast navigation. The selected individual in the learning phase is tested in the execution phase throughout exposing MR to intensive and complicated experiments. In this paper, the simulation is implemented using Matlab R2012a software.

\subsection{Learning phase}

The learning phase aims to find an individual in search space that has connections provided the best performance of MR. SARSA learns each individual in such a way that a connection in a judgment node among all available connections or an action in a processing node among all available actions is selected.

The performance of a GNP-RL is evaluated as follows. MR moves from the start point and chases the moving target in every trial. A 100 trails, $N=100$, were conducted, each trial contains a static and a dynamic obstacles. GNP$\mathrm{RL}$ is applied to control MR when it is in non-safe mode. The rate of steering angle change, $r(\Delta \theta)$, and the set point of speed, $v$, are used in calculating the fitness value in each step of non-safe mode. After applying GNP-RL on all trials, the fitness value of that individual is calculated using Eq. 7. These steps are repeated on all individuals in the population. The individual with highest fitness is selected as elite. The genetic operations are then applied to generate the next generation. Since then, the same steps of calculating the fitness values are applied to each individual to find the individual that has the highest fitness. In this paper, the total number of generations is 6000 , and the highest fitness value in each generation is shown in Fig. 7. It can be seen that the highest fitness value of the first generation is 2.06 , and this value is kept changed along all

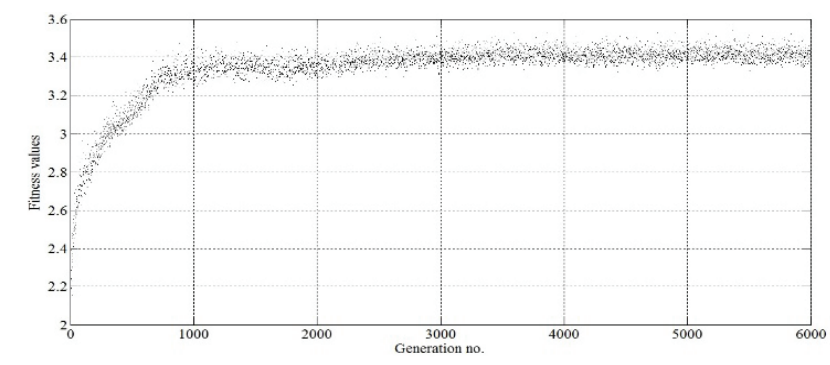

Fig. 7. Fitness values of GNP-RL generations until it stabilizes at 3.45 in the last generation. The individual which has the highest fitness value (elite) in the last generation is used later in the execution phase to measure its efficiency in providing the required safe navigation and smooth movement.

\subsection{Execution test}

Many experiments were conducted to test the efficiency of the proposed GNP-RL under wide variety of environment conditions in which many static and dynamic obstacles were used to disturb the MR movement. One of these experiments is explained as shown in Fig. 8. In this experiment, eight dynamic obstacles (d1-d8) that move randomly and eight static obstacles (s1-s8), divided into two groups, are located in the work space. In addition, the dynamic target (black color) is moving in upward exponentially sinusoidal form starting from [30 60 $]^{\mathrm{T}}$ with speed explained in Eq.8.

$$
v_{G}=\left[\begin{array}{c}
v_{x} \\
v_{y}
\end{array}\right]=\left[\begin{array}{c}
3 * \sin \left(\frac{G_{y}}{0.3}\right) e^{-0.02\left(G_{y}-90\right)} \\
0.15
\end{array}\right]
$$

The movements and locations of MR, dynamic obstacles, static obstacles, and the target are shown in Fig. 8a. MR speed is set to maximum, $0.75 \mathrm{~m} / \mathrm{s}$ when it is in safe mode (green color). But when MR becomes in the non-safe mode (red color), MR speed is controlled by GNP-RL when . Each dynamic obstacle (d1-d8) is moving in a certain constant speed, $0.5,0.4,0.5,0.5,0.6,0.7,0.35,0.7 \mathrm{~m} / \mathrm{s}$, respectively. Figs. $8 \mathrm{~b}-\mathrm{k}$ are expansions of the environment in obstacle avoidance occurrences to illustrate the details of MR steps at those moments. In this scenario, $d_{s}=2 m$, $d_{c}=0.3 m$, and $d_{w}=0.3 m$.

MR started its navigation at $\left[\begin{array}{ll}200 & 60\end{array}\right]^{\mathrm{T}}$ with maximum speed to chase the target and adjusted its turning angle towards the target until it encountered d1 at [197.75 60 $]^{\mathrm{T}}$ when it was in the fourth step as shown in Fig. 8b. At this moment MR controller was changed from the safe into non-safe mode. Since d1 crossed MR path, GNP-RL required 15 steps to the left to make MR exceeds this obstacle becomes in safe mode. This action helps MR to exceed $\mathrm{d} 1$ successfully, where minimum $d(M R, \mathrm{~d} 1)=$ $1.71 \mathrm{~m}$, with smooth path and satisfactory speed. After that, three and four steps were enough to make MR avoids collision with $\mathrm{d} 2$ and $\mathrm{d} 3$, respectively, as shown in Fig. 8c and Fig. 8d.

Fig. 8e shows the capability of MR under control of GNP-RL to exceed static obstacles. The maximum $\Delta \theta$ used in static obstacle avoidance is $34.97^{\circ}$, while it was around zero in all other steps. In Fig. 8f, d4 moved in the same direction of MR and crossed its path while it was moving upward left. Therefore, MR became in non-safe mode in step 98, and to avoid this obstacle it moved right 

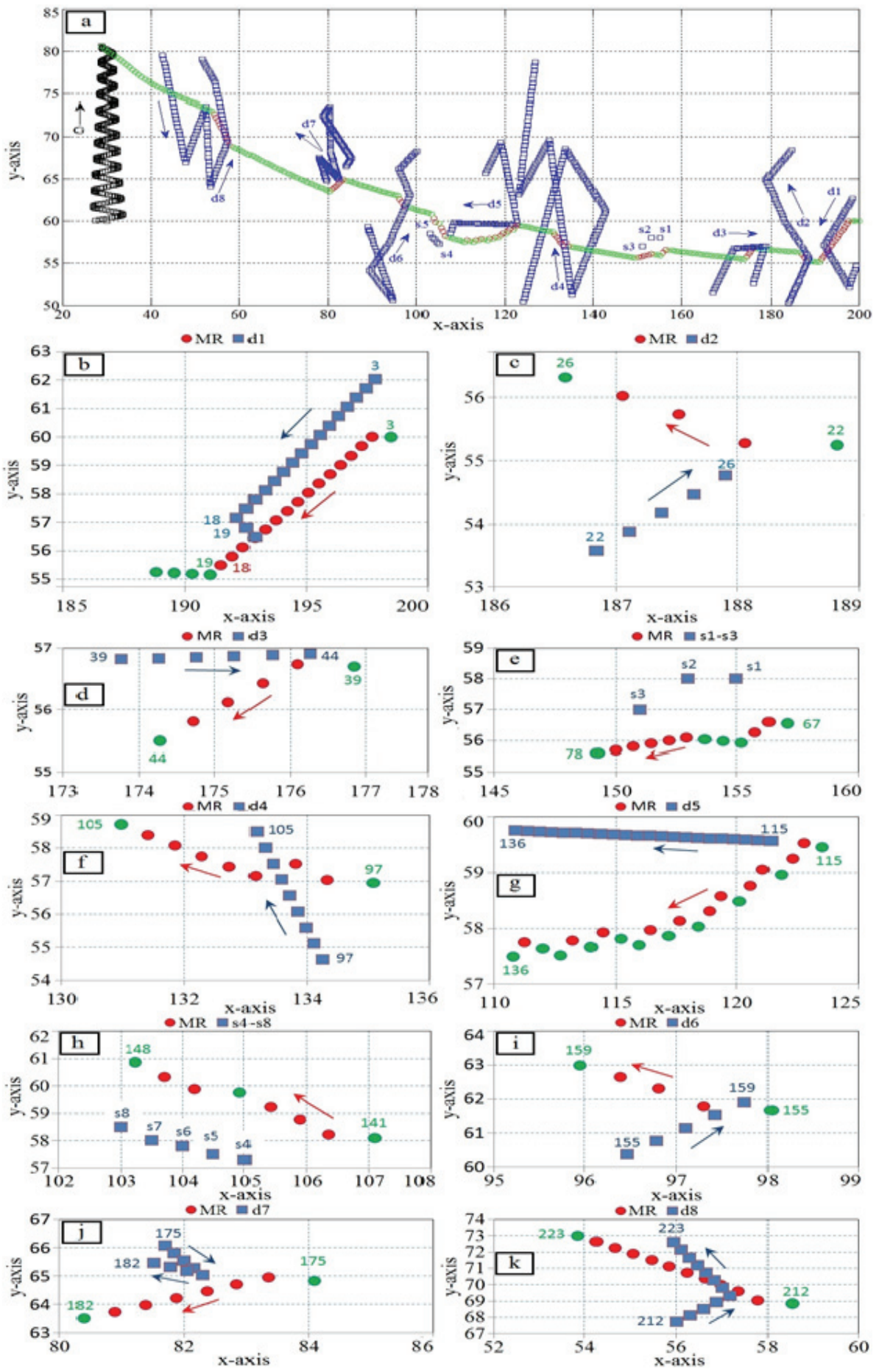

Fig. 8. Navigation in dynamic environment under GNP-RL control

and then left producing maximum $\Delta \theta=71.17^{\circ}$.

An example of a dynamic obstacle that is moving in parallel with the trajectory of MR and standing in the way between MR and target can be seen in Fig. $8 \mathrm{~g}$ in this example, many tries were done by MR to move toward target but it encountered $\mathrm{d} 5$ and entered non-safe region. Therefore, GNP-RL moved MR away of d5 to avoid this obstacle. This situation made MR switches between safe and non-safe regions for eight times until MR exceeded the effect of d5. After six steps, another group of static obstacles disturbed the navigation of MR in step 142 as shown in Fig. 8h, which were avoided by turning to the right with speed ranged between 0.66 and $0.74 \mathrm{~m} / \mathrm{s}$. After that, MR encountered d6 (Fig. 8i), which was moving upward right with speed $0.7 \mathrm{~m} / \mathrm{s}$. As a response of this facing, MR moved to the right for three steps where step 157 has maximum $\Delta \theta=37^{\circ}$.

In Fig's. $8 \mathrm{j}$ and $8 \mathrm{k}$, dynamic obstacles change their 

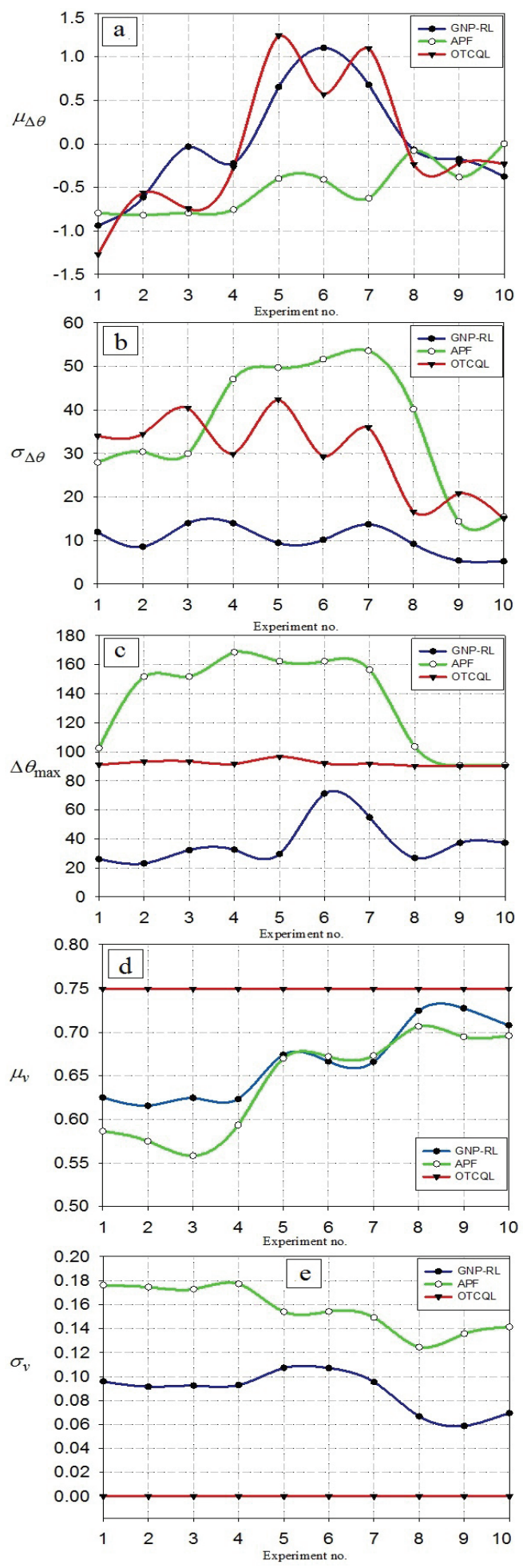

Fig. 9. Performance of MR under control of GNP-RL, OTCQL and APF: (a) Mean of $\Delta \theta\left(\mu_{\Delta \theta}\right)$; (b) Standard deviation of $\Delta \theta\left(\sigma_{\Delta \theta}\right)$; (c) Maximum $\Delta \theta\left(\Delta \theta_{\max }\right)$; (d) Mean of speed $\left(\mu_{v}\right)$; (e) Standard deviation of speed $\left(\sigma_{v}\right)$.

direction suddenly. In Fig $8 \mathrm{j}, \mathrm{d} 7$ changed its direction before it crossed the path of MR, while the direction change of $\mathrm{d} 8$ took place after it crossed MR path. No big steering angle has been used to avoid these obstacles where maximum $\Delta \theta=37.22^{\circ}$ took place in the first and last steps of each non-safe region, while small steering angles used in the following steps.

It can be seen that MR is capable of avoiding all of static obstacles successfully, though they are located close to each other. This proves that MR learned from the experiments in the learning phase such that it acquired the capability to avoid this kind of obstacles. Moreover, although the dynamic obstacles moved in different speeds, changed their directions randomly and suddenly, and encountered MR from different directions and angles, GNP-RL shows its efficiency to avoid these obstacles without using sharp turning angles.

\subsection{Performance evaluation of GNP-RL}

This subsection aims to conduct simulation to examine the efficiency and feasibility of the proposed GNP-RL compared with OTCQL [18] and APF [20]. Ten experiments were conducted and applied to each navigation scheme to measure their efficiency under same circumstances. In each experiment, the target is moved in either sinusoidal or exponentially sinusoidal form and started its movement from different positions in either direction, different number of dynamic obstacles with a random movement and a specified speed for each one, different number of static obstacles, and MR started its navigation from random positions. Fig. 9 shows the results of applying these three schemes on the ten experiments.

Referring to Fig. 9a, it can be noted that the mean value of steering angle change, $\mu_{\Delta \theta}$, is ranged between -1.269 and 1.244, which refers to the convergence of $\mu_{\Delta \theta}$ in all schemes under study, where APF shows the minimum $\mu_{\Delta \theta}$ in most of the conducted experiments. This small range of $\mu_{\Delta \theta}$ is due to the use of small steering angles during safe mode that are enough to chase the target but the big steering angles are used only during non-safe mode when MR encounters an obstacle.

Fig. $9 \mathrm{~b}$ shows the standard deviation of steering angle change, $\sigma_{\Delta \theta}$, for all the applied schemes and it can be seen that GNP-RL shows the minimum $\sigma_{\Delta \theta}$ among all the conducted experiments. That is, MR under control of GNPRL has the smoothest trajectory path than that of OTCQL and APF, where the difference between $\sigma_{\Delta \theta}$ of GNP-RL and that of OTCQL is varied between (7.45) and (32.96), and it varied between (8.99) and (41.49) compared with APF. Meanwhile, the maximum $\sigma_{\Delta \theta}$ in most of the applied experiments is taken place when MR under control of APF that refers to big variations of steering angle producing worst tortuous trajectory. The maximum angle difference, $\Delta \theta_{\max }$, used in every experiment for all the schemes under study proves also this fact. As shown in Fig. 9c, the largest steering angles are used by APF to exceed obstacles, while GNP-RL uses the minimum steering angle 
in all experiments, ranging between (23.04) and (71.17). This refers to the efficiency of GNP-RL to exceed same obstacles with minimum steering angle. Moreover, OTCQL uses fixed and large steering angle during obstacle avoidance resulting high $\sigma_{\Delta \theta}$ which refers to non-smooth navigation path (Fig. 9 a-c). However, OTCQL shows moderate $\Delta \theta$, ranging around $90^{\circ}$, between the performance of APF and GNP-RL. Consequently, large steering angles that have been used in APF and OTCQL cause a non-smooth trajectory of MR while GNP-RL provides the smoothest path during obstacle avoidance without the need to use sharp turns.

Since the speed of MR under OTCQL control is constant throughout the entire navigation process (Fig's. 9 d-e), it is assumed that the speed is set to its maximum value in these experiments. Therefore, MR under control of OTCQL moves in highest navigation speed than that of other schemes. GNP-RL shows its capability to drive MR in a reasonable speed ranges between 0.61 and $0.727 \mathrm{~m} / \mathrm{s}$ while maintaining its smooth movement. Moreover, GNP-RL shows low variation of speed $\left(\sigma_{v}=0.058,0.107\right)$, that is, MR changes its speed smoothly without the need for sudden changes. In contrast, APF drives MR with a slowest speed $\left(\mu_{v}=0.56,0.707\right)$ and highest variation of speed $\left(\sigma_{v}=0.124,0.177\right)$.

\subsection{Safe navigation test}

This subsection aims to examine the efficiency of GNPRL, compared with OTCQL and APF, in exceeding static and dynamic obstacles to satisfy safe navigation.

For this purpose, a simulation workspace is designed to ensure the existence of a facing between MR and at least one obstacle when it navigates in each trial. Every experiment of the designed workspace is composed of 200 trials. Each trial contains 12 dynamic obstacles and 12 static obstacles. The positions of these obstacles are initially predefined and remained fixed in all the trials. The dynamic obstacles are moving in a straight line without changing their direction during the execution time of each trial. But, the orientation angle of all obstacles is either incremented or decremented in each consecutive trial. Moreover, the movement direction of the target and the starting positions of MR and the target are chosen randomly in each trial, where the movement direction of the target is either up, down, right or left. If MR does not encounter an obstacle in any trial of the experiment, then the trial is repeated with another random selection of MR and target positions until such an encounter occurs. One of these trials is shown in Fig. 10, which represents the control of MR by GNP-RL in trial number 34 of one of the conducted experiment. MR, which moves to the right, exceeds two static obstacles (s3 and s4) and dynamic obstacle (d12) before catching the target which moves up.

Ten experiments were conducted to measure the degree of safe navigation of MR under the control of GNP-RL,

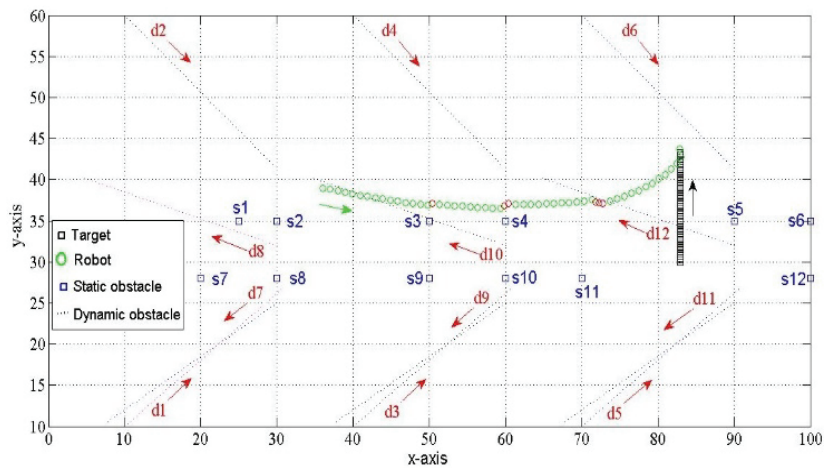

Fig. 10. Workplace trial of dynamic environment
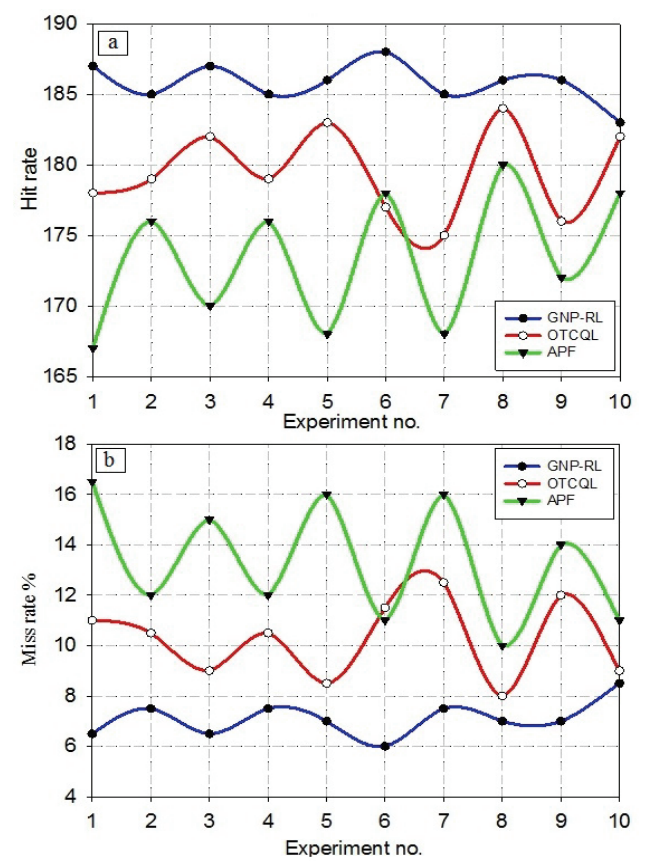

Fig. 11. Hit/Miss rates of GNP-RL, OTCQL, and APF algorithms: (a) Hit rate of GNP-RL; OTCQL and APF (b)

OTCQL, and APF where each experiment includes 200 trials. Hit and miss rates are used as a measure of efficiency of these algorithms. Hit rate can be defined as the frequency of the trials in which MR successes to catch the target without colliding with any obstacles. Accordingly, the miss rate is the frequency of the trials in which MR fails to catch the target. Fig. 11 shows the resulting hit/miss rates of applying these ten experiments.

The highest hit rate is provided by GNP-RL among the other algorithms where the lowest hit rate of GNP-RL (183) is almost equal or greater than the highest hit rate of OTCQL (184) and APF (180). This refers to the efficiency of GNP-RL in avoiding obstacles providing safest navigation comparing with the other algorithms under study, where its hit rate ranges between 183 and 188 . Although, MR under OTCQL presents fastest navigation, it is not efficient in exceeding obstacles, where its hit rate 
ranges between 175 and 184 , as well as its path is tortuous due to the large steering angles used to avoid obstacles. However, it provides a moderate safety ranges between GNP-RL and APF. On the other hand, APF presents lesser safety navigation than GNP-RL and OTCQL where its miss rate is almost higher than that of GNP-RL and OTCQL in all the conducted experiments.

\section{Conclusion}

This paper proposed a new formulation of GNP-RL for MR navigation in dynamic environment based on predicting the collision position. The dynamic environment encompasses static and dynamic obstacles as well as a moving target. The proposed Collision prediction of environment representation provides a satisfactory perception and prediction of the surrounding environment and its integration with judgment nodes of GNP-RL provides a suitable action for variant states of the dynamic environment. Efficiency and effectiveness of the proposed GNP-RL have been demonstrated through comparisons with OTCQL and APF, in terms of steering angle change, MR speed, and obstacles avoidance. Navigation results in different complex dynamic environments show the effectiveness of the proposed GNP-RL, where obstacles are avoided in safer, smoother, and satisfactory faster than other state-of-arts. In the future, replacing the evaluation of the surrounding environment in judgment nodes by fuzzy rules will be studied to provide better judgment and improve performance of GNP-RL.

\section{References}

[1] Zhang, Y., L. Zhang, and X. Zhang. Mobile Robot path planning base on the hybrid genetic algorithm in unknown environment. in Intelligent Systems Design and Applications, 2008. ISDA'08. Eighth International Conference on. 2008. IEEE.

[2] Belkhouche, F., Reactive path planning in a dynamic environment. Robotics, IEEE Transactions on, 2009. 25(4): p. 902-911.

[3] Du Toit, N.E. and J.W. Burdick, Robot motion planning in dynamic, uncertain environments. Robotics, IEEE Transactions on, 2012. 28(1): p. 101-115.

[4] Parhi, D.R., Navigation of mobile robots using a fuzzy logic controller. Journal of intelligent and robotic systems, 2005. 42(3): p. 253-273.

[5] Li, W. Fuzzy logic-basedperception-action'behavior control of a mobile robot in uncertain environments. in Fuzzy Systems, 1994. IEEE World Congress on Computational Intelligence., Proceedings of the Third IEEE Conference on. 1994. IEEE.

[6] Jaradat, M.A.K., M.H. Garibeh, and E.A. Feilat, Autonomous mobile robot dynamic motion planning using hybrid fuzzy potential field. Soft Computing, 2012. 16(1): p. 153-164.

[7] Mobadersany, P., S. Khanmohammadi, and S. Ghaemi. An efficient fuzzy method for path planning a robot in complex environments. in Electrical Engineering (ICEE), 2013 21st Iranian Conference on. 2013. IEEE.

[8] Mendonça, M., L.V.R. de Arruda, and F. Neves Jr, Autonomous navigation system using event drivenfuzzy cognitive maps. Applied Intelligence, 2012. 37(2): p. 175-188.

[9] Farooq, U., et al. A two loop fuzzy controller for goal directed navigation of mobile robot. in Emerging Technologies (ICET), 2012 International Conference on. 2012.

[10] Er, M. J. and C. Deng, Obstacle avoidance of a mobile robot using hybrid learning approach. Industrial Electronics, IEEE Transactions on, 2005. 52(3): p. 898-905.

[11] Hui, N.B. and D.K. Pratihar, A comparative study on some navigation schemes of a real robot tackling moving obstacles. Robotics and Computer-Integrated Manufacturing, 2009. 25(4): p. 810-828.

[12] Hui, N.B., V. Mahendar, and D.K. Pratihar, Timeoptimal, collision-free navigation of a car-like mobile robot using neuro-fuzzy approaches. Fuzzy Sets and Systems, 2006. 157(16): p. 2171-2204.

[13] Pratihar, D.K., K. Deb, and A. Ghosh, A genetic-fuzzy approach for mobile robot navigation among moving obstacles. International Journal of Approximate Reasoning, 1999. 20(2): p. 145-172.

[14] Dinham, M. and G. Fang. Time optimal path planning for mobile robots in dynamic environments. in Mechatronics and Automation, 2007. ICMA 2007. International Conference on. 2007. IEEE.

[15] Vukosavljev, S.A., et al. Mobile robot control using combined neural-fuzzy and neural network. in Computational Intelligence and Informatics (CINTI), 2011 IEEE 12th International Symposium on. 2011. IEEE.

[16] Singh, M.K., D.R. Parhi, and J.K. Pothal. ANFIS Approach for Navigation of Mobile Robots. in Advances in Recent Technologies in Communication and Computing, 2009. ARTCom'09. International Conference on. 2009. IEEE.

[17] Domínguez-López, J.A., et al., Adaptive neurofuzzy control of a robotic gripper with on-line machine learning. Robotics and Autonomous Systems, 2004. 48(2): p. 93-110.

[18] Kareem Jaradat, M.A., M. Al-Rousan, and L. Quadan, Reinforcement based mobile robot navigation in dynamic environment. Robotics and ComputerIntegrated Manufacturing, 2011. 27(1): p. 135-149.

[19] Ratering, S. and M. Gini, Robot navigation in a known environment with unknown moving obstacles. Autonomous Robots, 1995. 1(2): p. 149-165. 
[20] Ge, S.S. and Y.J. Cui, Dynamic motion planning for mobile robots using potential field method. Autonomous Robots, 2002. 13(3): p. 207-222.

[21] Sgorbissa, A. and R. Zaccaria, Planning and obstacle avoidance in mobile robotics. Robotics and Autonomous Systems, 2012. 60(4): p. 628-638.

[22] Agirrebeitia, J., et al., A new APF strategy for path planning in environments with obstacles. Mechanism and Machine Theory, 2005. 40(6): p. 645-658.

[23] Yaonan, W., et al., Autonomous mobile robot navigation system designed in dynamic environment based on transferable belief model. Measurement, 2011. 44(8): p. 1389-1405.

[24] Li, G., et al., Effective improved artificial potential field-based regression search method for autonomous mobile robot path planning. International Journal of Mechatronics and Automation, 2013. 3(3): p. 141170.

[25] Wilkie, D., J. van den Berg, and D. Manocha. Generalized velocity obstacles. in Intelligent Robots and Systems, 2009. IROS 2009. IEEE/RSJ International Conference on. 2009. IEEE.

[26] Chunyu, J., et al. Reactive target-tracking control with obstacle avoidance of unicycle-type mobile robots in a dynamic environment. in American Control Conference (ACC), 2010. 2010. IEEE.

[27] Mucientes, M., et al., Fuzzy temporal rules for mobile robot guidance in dynamic environments. Systems, Man, and Cybernetics, Part C: Applications and Reviews, IEEE Transactions on, 2001. 31(3): p. 391-398.

[28] Chang, C.C. and K.-T. Song, Environment prediction for a mobile robot in a dynamic environment. Robotics and Automation, IEEE Transactions on, 1997. 13(6): p. 862-872.

[29] Mabu, S., A. Tjahjadi, and K. Hirasawa, Adaptability analysis of genetic network programming with reinforcement learning in dynamically changing environments. Expert Systems with Applications, 2012. 39(16): p. 12349-12357.

[30] Sendari, S., S. Mabu, and K. Hirasawa. Fuzzy genetic Network Programming with Reinforcement Learning for mobile robot navigation. in Systems, Man, and Cybernetics (SMC), 2011 IEEE International Conference on. 2011. IEEE.

[31] Li, X., et al., Probabilistic Model Building Genetic Network Programming Using Reinforcement Learning. 2011. 2(1): p. 29-40.

[32] Mabu, S., et al. Evaluation on the robustness of genetic network programming with reinforcement learning. in Systems Man and Cybernetics (SMC), 2010 IEEE International Conference on. 2010. IEEE.

[33] Mabu, S., et al. Genetic Network Programming with Reinforcement Learning Using Sarsa Algorithm. in Evolutionary Computation, 2006. CEC 2006. IEEE Congress on. 2006. IEEE.
[34] Sendari, S., S. Mabu, and K. Hirasawa. Two-Stage Reinforcement Learning based on Genetic Network Programming for mobile robot. in SICE Annual Conference (SICE), 2012 Proceedings of. 2012. IEEE.

[35] Li, X., S. Mabu, and K. Hirasawa, Towards the maintenance of population diversity: A hybrid probabilistic model building genetic network programming. Trans. of the Japanese Society for Evol. Comput, 2010. 1(1): p. 89-101.

[36] Sutton, R.S. and A.G. Barto, Reinforcement learning: An introduction. Vol. 1. 1998: Cambridge Univ Press.

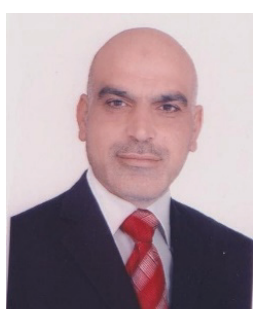

Ahmed H. M. Findi He received the B.Sc. and M.Sc. degrees in Control and Systems Engineering from the University of Technology, Iraq in 1990 and 1993 respectively. He is currently a Ph.D. student at the Electrical and Electronic Engineering Department, Faculty of Engineering, Universiti Putra Malaysia (UPM), Malaysia. His major research interests are intelligent control systems and autonomous mobile robots.

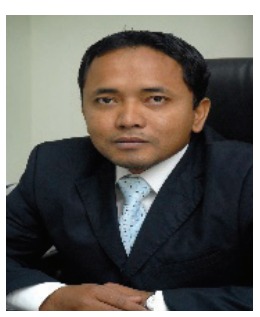

Mohammad H. Marhaban $\mathrm{He}$ received the B.Eng. degree in Electrical and Electronic Engineering from the University of Salford, UK, in 1998, and Ph.D. degree in Electronic Engineering from the University of Surrey, UK, in 2003. He is a Professor with the Department of Electrical and Electronic Engineering, Universiti Putra Malaysia. His research interests include intelligent control systems and computer vision.

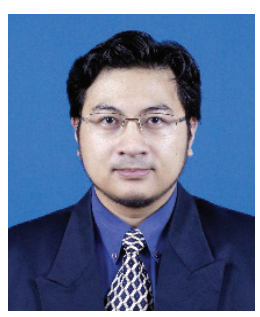

Raja Kamil He received the B.Eng. degree in electrical engineering from the University of Southampton, UK, and the Ph.D. degree in control engineering from the University of Sheffield, UK. He is now an Associate Professor with the Department of Electrical and Electronic Engineering, University Putra Malaysia, Malaysia and a Professional Engineer. His research interests include adaptive control, system identification and pattern recognition. 


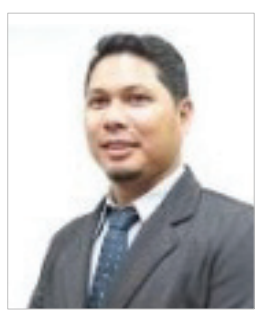

Mohd Khair Hassan He received his first degree in Electrical and Electronic from University of Portsmouth, UK in 1998, M.Eng Electrical Engineering degree from University of Technology, Malaysia in 2000 and completed his Ph.D in Automotive Engineering from Universiti Putra Malaysia in 2011. He is now an Associate Professor in Department of Electrical and Electronic Engineering, Faculty of Engineering, Universiti Putra Malaysia. $\mathrm{He}$ is also a Professional Engineer (PEng) in Electronic with the Board of Engineers Malaysia (BEM). His areas of interest include; control system, automotive control, and electric vehicle and AI applications. Currently, his research focuses on optimal strategy for energy consumption in electric vehicle and $\mathrm{x}-$ by-wire technology. 